\title{
A NOVEL FUZZY C-REGRESSION MODEL ALGORITHM USING A NEW ERROR MEASURE AND PARTICLE SWARM OPTIMIZATION
}

\author{
MoÊz SOLTANI，ABDELKADER CHAARI，FAYÇAL BEN HMIDA
}

Research Unit on Control, Monitoring and Safety of Systems (C3S)

High School of Sciences and Engineering of Tunis (ESSTT), 5, av. Taha Hussein, BP 56-1008 Tunis, Tunisia e-mail: soltani_c3s@yahoo.fr,\{assil.chaari,faycal.benhmida\}@esstt.rnu.tn

\begin{abstract}
This paper presents a new algorithm for fuzzy c-regression model clustering. The proposed methodology is based on adding a second regularization term in the objective function of a Fuzzy C-Regression Model (FCRM) clustering algorithm in order to take into account noisy data. In addition, a new error measure is used in the objective function of the FCRM algorithm, replacing the one used in this type of algorithm. Then, particle swarm optimization is employed to finally tune parameters of the obtained fuzzy model. The orthogonal least squares method is used to identify the unknown parameters of the local linear model. Finally, validation results of two examples are given to demonstrate the effectiveness and practicality of the proposed algorithm.
\end{abstract}

Keywords: Takagi-Sugeno fuzzy model, noise clustering algorithm, fuzzy c-regression model, orthogonal least squares, particle swarm optimization.

\section{Introduction}

In the past few years, fuzzy modeling algorithms have been widely used in many research areas because of their excellent ability of describing nonlinear systems. Fuzzy modeling is an effective tool for the approximation of uncertain systems on the basis of measured data (Hellendoorn and Driankov, 1997). The TakagiSugeno (T-S) model (Takagi and Sugeno, 1985) has been widely applied in many fields, such as modeling (Boukhris et al., 1999; Alci, 2008; Soltani et al., 2010a), control (Ying, 2000; Brdyś and Littler, 2002; Kościelny and Syfert, 2006; Qi and Brdys, 2009; Kluska, 2009) and fault tolerant control (Marx et al., 2007; Ichalal et al., 2010). In many studies, T-S based approaches such as the Gustafson-Kessel (GK) clustering algorithm (Gustafson and Kessel, 1979), the Gath-Geva (GG) algorithm (Gath and Geva, 1989), the fuzzy c-regression model clustering algorithm (Hathaway and Bezdek, 1993), enhanced fuzzy system models (Celikyilmaz and Burhan Turksen, 2008), the new FCRM clustering algorithm (NFCRMA) (Chaoshun et al., 2009; 2010) and the Fuzzy C-Means (FCM) clustering algorithm (Bezdek, 1981) are often used for the description of complex systems in a human intuitive way (especially the last one). A modified version of FCM, called the fuzzy c-regression model clustering algorithm, has been proposed by Hathaway and Bezdek (1993).

The FCRM algorithm develops hyper-plane-shaped clusters, while the FCM algorithm develops hyperspherical-shaped clusters. The FCRM algorithm suffers from two major problems:

- It uses an alternating optimization technique, whose iterative nature makes it sensitive to noise (Nasraoui and Krishnapuram, 1996; Sumit and Dave, 1998; Ichihashi and Honda, 2004; Leski, 2004). In addition, if the noisy data have significant high values, they can severely affect the model parameter estimation (Bezdek et al., 1999).

- Derived from Bezdek's fuzzy c-means algorithm, it is sensitive to initialization and may converge to a local minimum of the objective function (Frigui and Krishnapuram, 1999; Zhang et al., 2006; Xu and Zhang, 2009; Ying et al., 2011). Hence, different initializations may lead, easily, to different results.

The algorithms reported by Kim et al. (1998), Hathaway and Bezdek (1993), Chaoshun et al. (2009), Chaoshun et al. (2010), Chen et al. (1998), Rezaee and Zarandi (2010) as well as Andri and Ennu (2011) did not treat the two points cited above. Consequently, in order 
to deal with these two points, firstly we design a new objective function for a robust version of the FCRM algorithm based on the Noise Clustering (NC) algorithm (Dave, 1991). This latter has a robust capability against noise and is quite successful in improving the robustness of a variety of fuzzy clustering algorithms (Dave and Krishnapuram, 1997; Tran and Wagner, 1999; Ichihashi et al., 2005; Soltani et al., 2010b; Honda et al., 2010). Moreover, a new error measure is introduced into the objective function to get a good result. Secondly, many studies have proposed the evolutionary computation technique based on Particle Swarm Optimization (PSO). They have been successfully applied to solve various optimization problems (Zhang et al., 2006; 2011; Panchal et al., 2009; Niknam and Amiri, 2010). Thus, we introduce PSO into the FCRM clustering to achieve global optimization.

In this paper, new methods of the FCRM clustering algorithm are proposed using a modified objective function, a new error measure, and parameter estimation based particle swarm optimization. Based on these methods, we develop a fuzzy modeling approach for unknown nonlinear systems, in order to provide a good model approximation. The optimal consequent parameters of the local linear T-S model are estimated using the Orthogonal Least Squares (OLS) method.

The rest of this paper is organized as follows. In Section 2, a brief review of the FCRM clustering algorithm formulation is given, and in Section 3 the PSO algorithm is introduced. Three novel FCRM clustering algorithms are detailed in Section 4 considering the noise of the data set. Simulation results compared with other learning algorithms are shown in Section 5, and Section 6 summarizes the important features of our approach.

\section{Fuzzy c-regression model clustering algorithm}

A Takagi-Sugeno model consists of a set of fuzzy rules, each describing a local input-output relation as follows:

$$
\begin{aligned}
R_{i} & : \text { IF } x_{k 1} \text { is } A_{i 1} \text { and } \ldots \text { and } x_{k M} \text { is } A_{i M}, \\
\text { THEN } y_{i} & =a_{i 1} x_{k 1}+a_{i 2} x_{k 2}+\cdots+a_{i M} x_{k M}+b_{i 0}, \\
i & =1, \ldots, c
\end{aligned}
$$

where $R_{i}$ denotes the $i$-th IF-THEN rule, $A_{i j}$ is the fuzzy subset, and $c$ is the number of rules.

The affine T-S fuzzy model based on the FCRM belongs to the range of clustering algorithms with a linear prototype.

Let $S=\left\{\left(\mathbf{x}_{1}, y_{1}\right), \ldots,\left(\mathbf{x}_{N}, y_{N}\right)\right\}=\left\{\left(\mathbf{x}_{k}, y_{k}\right), k=\right.$ $1, \ldots, N\}$ be a set of input-output sample data pairs. Assume that the data pairs in $S$ are drawn from $c$ different fuzzy regression models. The hyper-plane of the $i$-th clus- ter representative is expressed as follows:

$$
\begin{aligned}
y_{k}= & f_{i}\left(\mathbf{x}_{k}, \boldsymbol{\theta}_{i}\right)+E_{i k}\left(\boldsymbol{\theta}_{i}\right), \\
= & a_{i 1} x_{k 1}+a_{i 2} x_{k 2}+\cdots+a_{i M} x_{k M} \\
& +b_{i 0}+E_{i k}\left(\boldsymbol{\theta}_{i}\right), \\
= & {\left[\mathbf{x}_{k} 1\right] \cdot \boldsymbol{\theta}_{i}^{T}+E_{i k}\left(\boldsymbol{\theta}_{i}\right), \quad i=1,2, \ldots, c, }
\end{aligned}
$$

where $\mathbf{x}_{k}=\left[x_{k 1}, \ldots, x_{k M}\right] \in \mathbb{R}^{M}$ is the input vector, $y_{k} \in \mathbb{R}$ is the output and $\boldsymbol{\theta}_{i}=\left[a_{i 1}, \ldots, a_{i M}, b_{i 0}\right] \in$ $\mathbb{R}^{M+1}$ is the parameter vector of the corresponding local linear model.

The distance (error measure) between the value predicted by the model $f_{i}\left(\mathbf{x}_{k}, \boldsymbol{\theta}_{i}\right)$ and the output $y_{k}$ is defined by

$$
E_{i k}\left(\boldsymbol{\theta}_{i}\right)=\left|y_{k}-\left[\begin{array}{ll}
\mathbf{x}_{k} & 1
\end{array}\right] . \boldsymbol{\theta}_{i}^{T}\right| .
$$

The distances $\left(E_{i k}\left(\boldsymbol{\theta}_{i}\right)\right)$ are weighted with the membership values $\mu_{i k}$ in the objective function that is minimized by the clustering algorithm and is given as

$$
J(S ; U, \boldsymbol{\theta})=\sum_{k=1}^{N} \sum_{i=1}^{c}\left(\mu_{i k}^{m}\right) E_{i k}^{2}\left(\boldsymbol{\theta}_{i}\right),
$$

where $m$ is the weighting exponent and $\mu_{i k}$ is the membership degree of $\mathbf{x}_{k}$ to the $i$-th cluster. The membership values $\mu_{i k}$ have to satisfy the following conditions:

$$
\begin{aligned}
& \mu_{i k} \in\left[\begin{array}{ll}
0 & 1
\end{array}\right], \quad i=1,2, \ldots, c, \quad k=1,2, \ldots, N, \\
& 0<\sum_{k=1}^{N} \mu_{i k}<N, \quad i=1,2, \ldots, c \\
& \sum_{i=1}^{c} \mu_{i k}=1, \quad k=1,2, \ldots, N .
\end{aligned}
$$

The identification procedure of the FCRM algorithm is summarized as follows (Hathaway and Bezdek, 1993). Given data $S$, set $m>1$ and specify regression models (Eqn. (2)), choose an error measure (Eqn. (3)). Select a termination threshold $\epsilon>0$ and initialize $U^{(0)}$ (e.g., at random).

Repeat for $l=1,2, \ldots$.

Step 1. Calculate values for $c$ model parameters $\boldsymbol{\theta}_{i}^{(l)}$ in Eqn. (2) that globally minimize the restricted function Eqn. (4).

Step 2. Update $U^{(l)}$ with $E_{i k}\left(\boldsymbol{\theta}_{i}^{(l)}\right)$, to satisfy

$$
= \begin{cases}U_{i k}^{(l)}\left[\sum_{j=1}^{c}\left(\frac{E_{i k}}{E_{j k}}\right)^{\frac{2}{m-1}}\right]^{-1} & \text { if } E_{i k}>0 \text { for } 1 \leq i \leq c, \\ 0 & \text { otherwise. }\end{cases}
$$

Until $\left\|U^{(l)}-U^{(l-1)}\right\| \leq \epsilon$, then stop. Otherwise, set $l=l+1$ and return to Step 1 . 


\section{New FCRM clustering algorithm}

Several authors have shown that the clustering results can be severely distorted when they are based on noisy data (Ohashi, 1984; Chen and Wang, 1999; Kim et al., 2004; Yang et al., 2005). To overcome this problem, many studies on the robust fuzzy modeling technique have been reported, one of them being the NC algorithm. In this approach, noise is considered a separate class. It is represented by a fictitious prototype that has a constant distance $\delta$ from all the data points. The membership $\mu_{* k}$ of point $x_{k}$ in the noise cluster is given by

$$
\mu_{* k}=1-\sum_{i=1}^{c} \mu_{i k} .
$$

Thus, the membership constraint for the good clusters is effectively relaxed to

$$
\sum_{i=1}^{c} \mu_{i k}<1
$$

Dave's objective function is given by

$$
\begin{aligned}
& J_{N C}(U, V) \\
& =\sum_{i=1}^{c} \sum_{k=1}^{N} \mu_{i k}^{m} D_{i k}^{2}+\sum_{k=1}^{N} \delta^{2}\left(1-\sum_{i=1}^{c} \mu_{i k}\right)^{m},
\end{aligned}
$$

for any input $\mathbf{x}_{k}$ in subspace $i$ denoted by center $\mathbf{v}_{i}, D_{i k}=$ $\left\|\mathbf{x}_{k}-\mathbf{v}_{i}\right\|$.

The combination of the noise clustering algorithm with the FCRM algorithm can lead to a new FCRM objective function as follows:

$$
\begin{aligned}
J_{\text {new }} & (S ; U, \boldsymbol{\theta}) \\
= & \sum_{k=1}^{N} \sum_{i=1}^{c} \mu_{i k}^{m} E_{i k}^{2}\left(\boldsymbol{\theta}_{i}\right)+\sum_{k=1}^{N} \delta^{2} \mu_{* k}^{m} .
\end{aligned}
$$

In Eqn. (12), the first term on the left hand side is the same as the original FCRM objective function, while the second term is due to the noise clustering extension. Here $\delta$ is a scale parameter and may be used based on the idea presented by Dave (1991) as

$$
\delta^{2}=\gamma \frac{1}{c N} \sum_{k=1}^{N} \sum_{i=1}^{c} E_{i k}^{2}\left(\boldsymbol{\theta}_{i}\right),
$$

where $\gamma$ is a user-defined parameter depending on the example type.

To solve the constrained problem $J_{\text {new }}$ with respect to $\mu_{i k}$, we introduce $N$ Lagrange multipliers $\lambda_{k}, k=$ $1, \ldots, N$. The minimization of $J_{\text {new }}$ starts by forming the Lagrangian

$$
F=J_{\text {new }}-\sum_{k=1}^{N} \lambda_{k}\left(\sum_{i=1}^{c} \mu_{i k}+\mu_{* k}-1\right) .
$$

By differentiating the Lagrangian with respect to $\mu_{i k}$, $\mu_{* k}$ and $\lambda_{k}$ and setting the derivatives to zero, we obtain

$$
\begin{gathered}
\frac{\partial F}{\partial \mu_{i k}}=m \mu_{i k}^{m-1} E_{i k}^{2}-\lambda_{k}=0, \\
\frac{\partial F}{\partial \mu_{* k}}=m \delta^{2} \mu_{* k}^{m-1}-\lambda_{k}=0, \\
\frac{\partial F}{\partial \lambda_{k}}=\sum_{i=1}^{c} \mu_{i k}+\mu_{* k}-1=0 .
\end{gathered}
$$

From Eqns. (15) and (16), we get

$$
\mu_{i k}=\left[\frac{\lambda_{k}}{m}\right]^{\frac{1}{m-1}}\left[\frac{1}{E_{i k}^{2}}\right]^{\frac{1}{m-1}}
$$

and

$$
\mu_{* k}=\left[\frac{\lambda_{k}}{m}\right]^{\frac{1}{m-1}}\left[\frac{1}{\delta^{2}}\right]^{\frac{1}{m-1}}
$$

Using Eqns. (17)-(19), we get

$$
\left[\frac{\lambda_{k}}{m}\right]^{\frac{1}{m-1}}=\frac{1}{\sum_{j=1}^{c}\left(\frac{1}{E_{j k}}\right)^{\frac{2}{m-1}}+\left(\frac{1}{\delta}\right)^{\frac{2}{m-1}}}
$$

and then, by substituting this into Eqn. (18), the following equation can be obtained:

$$
\mu_{i k}=\frac{1}{\sum_{j=1}^{c}\left(\frac{E_{i k}}{E_{j k}}\right)^{\frac{2}{m-1}}+\left(\frac{E_{i k}}{\delta}\right)^{\frac{2}{m-1}}} .
$$

From Eqns. (2) and (12), the objective function of the New FCRM (NFCRM) clustering algorithm is defined as

$$
\begin{aligned}
J_{\text {new }}(S ; U, \boldsymbol{\theta})= & \sum_{k=1}^{N} \sum_{i=1}^{c} \mu_{i k}^{m}\left(y_{k}-\left[\begin{array}{ll}
\mathbf{x}_{k} & 1
\end{array}\right] \boldsymbol{\theta}_{i}^{T}\right)^{2} \\
& +\sum_{k=1}^{N} \delta^{2}\left(1-\sum_{i=1}^{c} \mu_{i k}\right)^{m} \\
= & \sum_{k=1}^{N} \sum_{i=1}^{c} \mu_{i k}^{m}\left(y_{k}-\sum_{j=1}^{M+1} \theta_{i j} \hat{x}_{k j}\right)^{2} \\
& +\sum_{k=1}^{N} \delta^{2}\left(1-\sum_{i=1}^{c} \mu_{i k}\right)^{m}
\end{aligned}
$$

where $\hat{x}_{k}=\left[\begin{array}{ll}\mathbf{x}_{k} & 1\end{array}\right]$ and $\hat{x}_{k j}$ is $j$-th coordinate of the vector $\left[\begin{array}{ll}\mathbf{x}_{k} & 1\end{array}\right]$.

The partial derivative of the objective function in Eqn. (22) is

$$
\frac{\partial J_{\text {new }}}{\partial \theta_{i j}}=-2 \sum_{k=1}^{N} \mu_{i k}^{m}\left(y_{k}-\sum_{t=1}^{M+1} \theta_{i t} \hat{x}_{k t}\right) \hat{x}_{k j},
$$


and then

$$
\begin{gathered}
\theta_{i j}=\frac{\sum_{k=1}^{N} \mu_{i k}^{m}\left(y_{k}-\sum_{t \neq j} \theta_{i t} \hat{x}_{k t}\right) \hat{x}_{k j}}{\sum_{k=1}^{N} \mu_{i k}^{m} \hat{x}_{k j}^{2}}, \\
i=1,2, \ldots, c, \quad j=1,2, \ldots, M+1 .
\end{gathered}
$$

Based on the optimization conditions (Eqns. (21) and (24)), the identification algorithm for Type 1 NFCRM (NFCRM1) via iterative optimization is given as follows (Soltani et al., 2011).

\section{Algorithm: NFCRM1}

Given a data $S$, set $m>1$. Fix $\gamma>0$ and the parameter vectors $\boldsymbol{\theta}_{i}$ at random. Pick a termination threshold $\epsilon>0$ and an initial partition $U^{(0)}$.

Repeat for $l=1,2, \ldots$.

Step 1. Compute error measure $E_{i k}\left(\boldsymbol{\theta}_{i}\right)$ via Eqn. (3). Step 2. Calculate $\delta^{2}$ via Eqn. (13).

Step 3. Compute $\mu_{i k}^{(l)}$ and $\theta_{i j}^{(l)}$ via Eqns. (21) and (24), respectively.

Step 4. Compute err $=\left\|U^{(l)}-U^{(l-1)}\right\|$.

Until err $\leq \epsilon$, then stop. Otherwise, set $l=l+1$ and return to Step 1 .

As mentioned by Wu and Yang (2002), the nonEuclidean distance is more robust than the Euclidean one. Then we give an extension of the non-Euclidean distance in the case of the NFCRM1 algorithm. By transforming (Eqn. (3)), the new error measure of is defined as

$$
E_{i k}\left(\boldsymbol{\theta}_{i}\right)=\sqrt{1-\exp \left(-\rho\left|y_{k}-\left[\mathbf{x}_{k} 1\right] \cdot \boldsymbol{\theta}_{i}^{T}\right|\right)}
$$

where $\rho$ is a positive constant. Then the NFCRM1 objective function (Eqn. (12)) is rewritten as follows:

$$
\begin{aligned}
J_{\text {new }}(S ; U, \boldsymbol{\theta})= & \sum_{k=1}^{N} \sum_{i=1}^{c} \mu_{i k}^{m} E_{i k}^{2}\left(\boldsymbol{\theta}_{i}\right) \\
& +\sum_{k=1}^{N} \delta^{2}\left(1-\sum_{i=1}^{c} \mu_{i k}\right)^{m} .
\end{aligned}
$$

Equations (21) and (13) can be respectively rewritten as

$$
\mu_{i k}=\frac{1}{\sum_{j=1}^{c}\left(\frac{E_{i k}}{E_{j k}}\right)^{\frac{2}{m-1}}+\left(\frac{E_{i k}}{\delta}\right)^{\frac{2}{m-1}}}
$$

and

$$
\delta^{2}=\gamma \frac{1}{c N} \sum_{k=1}^{N} \sum_{i=1}^{c} E_{i k}^{2}\left(\boldsymbol{\theta}_{i}\right)
$$

\section{Algorithm: NFCRM2}

Fix $\rho>0, \gamma>0$ and choose parameter vector $\boldsymbol{\theta}_{i}$ at random. Set a termination threshold $\epsilon>0$ and an initial partition $U^{(0)}$.

Repeat for $l=1,2, \ldots$.

Step 1. Compute error measure $E_{i k}\left(\boldsymbol{\theta}_{i}\right)$ via Eqn. (25).

Step 2. Calculate $\delta^{2}$ via Eqn. (28).

Step 3. Compute $\mu_{i k}^{(l)}$ and $\theta_{i j}^{(l)}$ via Eqn. (27) and Weighted Recursive Least-Squares (WRLS), respectively.

Step 4. Compute err $=\left\|U^{(l)}-U^{(l-1)}\right\|$.

Until err $\leq \epsilon$, then stop. Otherwise set $l=l+1$ and return to Step 1.

The algorithm based new error measure is called Type 2 NFCRM (NFCRM2).

Two NFCRM algorithms are presented and developed taking into account the noisy data. The random initialization leads to the convergence to a local minimum of the objective function. To overcome this problem, we introduce PSO into the NFCRM2 algorithm to achieve global optimization.

\section{NFCRM based on PSO (PSO-NFCRM)}

4.1. PSO algorithm. Particle swarm optimization was first introduced by Kennedy and Eberhat (1995). The PSO algorithm has been successfully applied to solve various optimization problems. Panchal et al. (2009) proposed PSO based clustering algorithms for remote image classification. A Multi-swarm Cooperative PSO (MCPSO) was used to adjust the parameters of the T-S fuzzy model and for the control of nonlinear dynamical systems (Ben et al., 2008). Qiang and Xinjian (2011) proposed a PSObased FCM clustering algorithm encoded by membership in order to handle data sets with dimensions smaller than the number of samples. Liang et al. (2009) used a PSO algorithm to optimize the initial clustering centers of the possibilistic c-means algorithm for image segmentation.

The PSO algorithm is initialized with a population of random solutions, called particles, to find an optimization result. Each particle has a position and a velocity, representing a possible solution to the optimization problem and a search direction in the search space. In each iterative process, the particle adjusts the velocity and position according to the best experience called the pbest, found by itself, and gbest, found by all its neighbors (Liang et al., 2009). For every generation, the velocity and po- 
sition can be updated by the following equations:

$$
\begin{aligned}
\mathbf{V}_{p d}^{k+1}= & \omega \mathbf{V}_{p d}^{k}+c_{1} r_{1}\left(\mathbf{p b e s t}^{k}-\mathbf{X}_{p d}^{k}\right) \\
& +c_{2} r_{2}\left(\mathbf{g b e s t}^{k}-\mathbf{X}_{i d}^{k}\right) \\
\mathbf{X}_{p d}^{k+1}= & \mathbf{X}_{i d}^{k}+\mathbf{V}_{p d}^{k+1}
\end{aligned}
$$

where $\omega$ is the inertia weight, which often changes from 0.2 to $0.9 ; k$ is the iteration number; $\mathbf{V}_{p d}^{k}$ is the velocity in the $d$-th dimension of the $p$-th particle; $\mathbf{x}_{i d}^{k}$ is the position in the $d$-th dimension of the $p$-th particle; pbest and gbest are the memory of the particle; $c_{1}$ and $c_{2}$ are the cognition and the social factor, respectively; $r_{1}$ and $r_{2}$ are random functions uniformly distributed in $\left[\begin{array}{ll}0 & 1\end{array}\right]$.

4.2. PSO-NFCRM algorithm. The PSO-NFCRM algorithm combines the advantages of the new fuzzy cregression model clustering algorithm of Type 2 and the PSO algorithm. To evaluate each particle, the fitness function is defined as follows:

$$
\text { Fitness }=\frac{G}{J_{\text {new }}(S ; U, \boldsymbol{\theta})},
$$

where $G$ is a user-defined parameter.

The PSO-NFCRM clustering algorithm is summarized in 7 steps.

\section{Algorithm: PSO-NFCRM}

Fix $\rho>0, \gamma>0$ and choose parameter vectors $\boldsymbol{\theta}_{i}$ at random. Select a termination threshold $\epsilon>0$ and an initial partition $U^{(0)}$. Choose the number of particles $N_{P}$; Initialize the position and velocity of each particle, fix learning factors $c_{1}$ and $c_{2}$ and the inertia weight $\omega$.

Repeat for $l=1,2, \ldots$.

Step 1. Compute error measure $E_{i k}\left(\boldsymbol{\theta}_{i}\right)$ via Eqn. (25).

Step 2. Calculate $\delta^{2}$ via Eqn. (28).

Step 3. Compute $\mu_{i k}^{(l)}$ and $\theta_{i j}^{(l)}$ via Eqn. (27) and WRLS, respectively.

Step 4. Calculate the fitness value of each particle according to Eqn. (31).

Step 5. Find the individual best pbest for each particle and the global best gbest.

Step 6. Update the velocity and the position of each particle using Eqns. (29) and (30), respectively.

Step 7. Compute err $=\left\|\mathbf{V}^{(l)}-\mathbf{V}^{(l-1)}\right\|$.

Until err $\leq \epsilon$, then stop. Otherwise, set $l=l+1$ and return to Step 1.

4.3. Estimation of antecedent and consequent parameters. We use the novel fuzzy c-regression models for decomposition of the input-output space into multiple linear structures. Gaussian membership functions are usually chosen to represent the fuzzy sets in the premise part of each fuzzy rule. As mentioned by Hathaway and Bezdek (1993) as well as Chaoshun et al. (2009), the antecedent parameters can be easily obtained using $\mu_{i k}$. The fuzzy sets centers $\nu_{i k}$ and the standard deviations $\sigma_{i k}$ are calculated as follows:

$$
\begin{aligned}
& \nu_{i j}=\frac{\sum_{k=1}^{N} \mu_{i k} x_{k j}}{\sum_{k=1}^{N} \mu_{i k}}, \\
& \quad i=1,2, \ldots, c, \quad j=1,2, \ldots, M,
\end{aligned}
$$

$$
\sigma_{i j}=\sqrt{\frac{2 \sum_{k=1}^{N} \mu_{i k}\left(x_{k j}-\nu_{i j}\right)^{2}}{\sum_{k=1}^{N} \mu_{i k}}} .
$$

Once the antecedent parameters have been fixed, the OLS method (Chen et al., 1989; Wu et al., 2005) can be applied to estimate the consequent parameters for each rule. Using OLS, the consequent parameters are estimated by transforming the model (1) into an equivalent auxiliary one

$$
\mathbf{Y}=P \boldsymbol{\Theta}+\mathbf{e},
$$

where $\mathbf{Y}=\left[y_{1}, \ldots, y_{N}\right]^{T}, P=\left[\mathbf{p}_{1}, \ldots, \mathbf{p}_{M}\right]$ with $\mathbf{p}_{i}=\left[p_{i}\left(x_{1}\right), \ldots, p_{i}\left(x_{N}\right)\right], \boldsymbol{\Theta}=\left[\Theta_{1}, \ldots, \Theta_{M}\right]^{T}$ and $\mathbf{e}=\left[e_{1}, \ldots, e_{N}\right]^{T}$.

The OLS algorithm is described as follows (Wang and Mendel, 1992):

Step 1. For $1 \leq i \leq M$, compute

$$
\begin{gathered}
\mathbf{w}_{1}^{(i)}=\mathbf{p}_{i}, g_{1}^{(i)}=\frac{\left(\mathbf{w}_{1}^{(i)}\right)^{T} \mathbf{Y}}{\left(\mathbf{w}_{1}^{(i)}\right)^{T} \mathbf{w}_{1}^{(i)}} \\
{[\mathrm{err}]_{1}^{(i)}=\frac{\left(g_{1}^{(i)}\right)^{2}\left(\mathbf{w}_{1}^{(i)}\right)^{T} \mathbf{w}_{1}^{(i)}}{\mathbf{Y}^{T} \mathbf{Y}} .}
\end{gathered}
$$

Find

$$
[\mathrm{err}]_{1}^{\left(i_{1}\right)}=\max \left([\mathrm{err}]_{1}^{(i)}\right)
$$

and select

$$
\mathbf{w}_{1}=\mathbf{w}_{1}^{\left(i_{1}\right)}=\mathbf{p}_{\left(i_{1}\right)}, \quad g_{1}=g_{1}^{\left(i_{1}\right)}
$$

Step 2. For $2 \leq i \leq M$, for $1 \leq k \leq N, i \neq i_{1}, \ldots, i \neq$ $i_{k}$, compute 


$$
\begin{aligned}
\alpha_{j k}^{(i)} & =\frac{\mathbf{w}_{j}^{T} \mathbf{p}_{i}}{\mathbf{w}_{j}^{T} \mathbf{w}_{j}}, \quad 1 \leq j<i, \\
\mathbf{w}_{k}^{(i)} & =\mathbf{p}_{i}-\sum_{j=1}^{k-1} \alpha_{j k}^{(i)} \mathbf{w}_{j}, \\
g_{k}^{(i)} & =\frac{\left(\mathbf{w}_{k}^{(i)}\right)^{T} \mathbf{Y}}{\left(\mathbf{w}_{k}^{(i)}\right)^{T} \mathbf{w}_{k}^{(i)}}, \\
{[\operatorname{err}]_{k}^{(i)} } & =\frac{\left(g_{k}^{(i)}\right)^{2}\left(\mathbf{w}_{k}^{(i)}\right)^{T} \mathbf{w}_{k}^{i}}{\mathbf{Y}^{T} \mathbf{Y}} .
\end{aligned}
$$

Find

$$
[\operatorname{err}]_{k}^{\left(i_{k}\right)}=\max \left([\operatorname{err}]_{k}^{(i)}\right)
$$

and select

$$
\mathbf{w}_{k}=\mathbf{w}_{k}^{\left(i_{k}\right)}, \quad g_{k}=g_{k}^{\left(i_{k}\right)} .
$$

Step 3. Solve the triangular system $A \boldsymbol{\Theta}=\mathbf{g}$, where

$$
\begin{aligned}
A & =\left(\begin{array}{ccccc}
1 & \alpha_{12}^{i_{2}} & \alpha_{13}^{i_{3}} & \cdots & \alpha_{1 M}^{i_{M}} \\
0 & 1 & \alpha_{23}^{2} & \cdots & \alpha_{2 M}^{i_{M}} \\
\vdots & \ddots & \ddots & \cdots & \vdots \\
\vdots & \cdots & \ddots & 1 & \alpha_{M-1, M}^{i_{M}} \\
0 & 0 & \cdots & 0 & 1
\end{array}\right), \\
\mathbf{g} & =\left[g_{1}, \cdots, g_{M}\right]^{T} .
\end{aligned}
$$

\section{Simulation results}

In this section, we are going to examine the performance of the proposed clustering algorithms developed above.

In this paper, the Mean Square Error (MSE) is used as the Performance Index (PI), which is defined as

$$
\operatorname{MSE}=\frac{1}{N} \sum_{k=1}^{N}\left(y_{k}-\hat{y}_{k}\right)^{2} .
$$

5.1. Benchmark problem. We consider the nonlinear system given as (Bidyadhar and Debashisha, 2011)

$$
y_{k}=\frac{y_{k-1}\left(y_{k-2}+2\right)\left(y_{k-1}+2.5\right)}{8.5+y_{k-1}^{2}+y_{k-2}^{2}}+u_{k}+v_{k}
$$

which is used as a test for identification techniques introduced in this paper, to demonstrate the effectiveness of the proposed algorithms in a noisy environment. Here $y_{k}$ is the output, $u_{k}$ is the input which is uniformly bounded in the region $[-1,1]$ and $v_{k}$ is a white noise with zero mean and variance $\sigma^{2}$, which is added to the output system at different SNR levels (SNR $=1,5,10,15$ and $20 \mathrm{~dB}$ ).

We simulated two experimental cases: Case 1 and Case 2. The training data set contains 500 input-output pairs while for the testing 1000 data pairs are generated by the following input signal:

$u_{k}=\left\{\begin{aligned} \sin \left(\frac{2 k \pi}{250}\right) & \text { if } k<=500, \\ 0.8 \sin \left(\frac{2 k \pi}{250}\right)+0.2 \sin \left(\frac{2 k \pi}{25}\right) & \text { otherwise. }\end{aligned}\right.$

Tables 1-6 compare our results with those obtained with different algorithms such as Gustafson-Kessel (GK) (Gustafson and Kessel, 1979), the New FCRM Algorithm (NFCRMA) (Chaoshun et al., 2009), FCM (Hoppner et al., 1999) and the Fuzzy Model Identification (FMI) clustering algorithm (Chen et al., 1998). We choose $\{y(k-1), y(k-2), u(k), u(k-1)\}$ as input variables, and the number of fuzzy rules is four. The parameter settings are $\gamma=0.1$ and $\{\gamma=0.01, \rho=0.1\}$ for the NFCRM1 and NFCRM2 algorithms, respectively. In addition, the PSO-NFCRM algorithm performs best under the following settings: $\omega=0.94, G=1, c_{1}=c_{2}=2, N_{P}=50$. In Case 1, we compare our results with those cited above

Table 1. Comparison results (Case 1).

\begin{tabular}{|c|c|c|}
\hline Algorithms & MSE $_{\operatorname{Tr}}$ & MSE $_{\mathrm{Ts}}$ \\
\hline \hline FCM & 0.0090 & 0.2220 \\
GK & 0.0046 & 0.1347 \\
FMI & 0.0013 & 0.0181 \\
NFCRMA & $5.20 \mathrm{e}-4$ & 0.0096 \\
NFCRM1 & $4.76 \mathrm{e}-4$ & 0.0052 \\
NFCRM2 & $3.94 \mathrm{e}-4$ & 0.0045 \\
PSO-NFCRM & $1.80 \mathrm{e}-4$ & 0.0020 \\
\hline
\end{tabular}

with regard to the noisy data. Table 1 shows the various modeling performance results obtained by different algorithms. MSE ${ }_{T r}$ and $\mathrm{MSE}_{\mathrm{Ts}}$ are the MSE for training and testing data, respectively. The comparison results demonstrate that the best MSE is obtained by the proposed methods. In the absence of noise, the positive scalar parameter $\delta$ can be regarded as a regulatory factor to reduce the sensitivity of the model to the identification data.

In Case 2, the noise influence is analyzed with different SNR levels $(\mathrm{SNR}=1,5,10,15$ and $20 \mathrm{~dB})$. The parameter settings are: $\gamma=0.1$ and $\{\gamma=0.1, \rho=1\}$ for the NFCRM1 and NFCRM2 algorithms, respectively. In addition, the PSO-NFCRM algorithm performs best under the following settings: $\omega=0.9, G=1, c_{1}=c_{2}=2$, $N_{P}=50 . \quad$ As shown in Tables 2-6 both algorithms (FCRM1 and FCRM2) present almost similar performance for the lower level of noise regarding Figs. 2 and 3. However, only the PSO-FCRM algorithm retained good performance with a higher level of noise. On the whole, we 
Table 2. Comparison results with $S N R=20 \mathrm{~dB}$ (Case 2).

\begin{tabular}{|c|c|c|}
\hline Algorithms & MSE $_{T r}$ & MSE $_{T s}$ \\
\hline \hline FCM & 0.0285 & 0.2417 \\
GK & 0.0258 & 0.1867 \\
FMI & 0.0134 & 0.0802 \\
NFCRMA & 0.0133 & 0.0621 \\
NFCRM1 & 0.0126 & 0.0473 \\
NFCRM2 & 0.0116 & 0.0442 \\
PSO-NFCRM & 0.0107 & 0.0321 \\
\hline
\end{tabular}

Table 3. Comparison results with $S N R=15 \mathrm{~dB}$ (Case 2).

\begin{tabular}{|c|c|c|}
\hline Algorithms & MSE $_{\text {Tr }}$ & MSE $_{\text {Ts }}$ \\
\hline \hline FCM & 0.0533 & 0.3164 \\
GK & 0.0471 & 0.2212 \\
FMI & 0.0373 & 0.1110 \\
NFCRMA & 0.0363 & 0.0806 \\
NFCRM1 & 0.0355 & 0.0786 \\
NFCRM2 & 0.0342 & 0.0770 \\
PSO-NFCRM & 0.0322 & 0.0732 \\
\hline
\end{tabular}

Table 4. Comparison results with $S N R=10 \mathrm{~dB}$ (Case 2).

\begin{tabular}{|c|c|c|}
\hline Algorithms & MSE $_{\mathrm{Tr}}$ & MSE $_{\mathrm{Ts}}$ \\
\hline \hline FCM & 0.1155 & 0.5505 \\
GK & 0.1100 & 0.3042 \\
FMI & 0.1068 & 0.2136 \\
NFCRMA & 0.1039 & 0.1926 \\
NFCRM1 & 0.0963 & 0.1836 \\
NFCRM2 & 0.0947 & 0.1762 \\
PSO-NFCRM & 0.0913 & 0.1683 \\
\hline
\end{tabular}

note that, whatever the noise level is, our proposed algorithms always keep the best performance (Figs. 2 and 3). As shown in Fig. 1, our algorithms give the best accuracy models compared with other existing algorithms in the literature. Thus, they are more robust to noise, especially the PSO-NFCRM algorithm.

Figure 1(a) shows original and the identified data obtained using FCM for the testing data set, and Fig. 1(b) presents the respective errors. Figures 1(c) and (e) show the original and the identified data obtained using the NFCRMA and PSO-NCFRM algorithms for the testing data set, and Figs. 1(d) and (f) present the respective errors.

5.2. Box-Jenkins system. We consider the BoxJenkins gas furnace data set (Box and Jenkins, 1970), which is used as a standard test for identification techniques. The data set consists of 296 pairs of input-output measurements. The input $u$ is the gas flow rate into a fur-
Table 5. Comparison results with $S N R=5 \mathrm{~dB}$ (Case 2).

\begin{tabular}{|c|c|c|}
\hline Algorithms & MSE $_{\operatorname{Tr}}$ & MSE $_{\mathrm{Ts}}$ \\
\hline \hline FCM & 0.4577 & 0.8167 \\
GK & 0.3364 & 0.8094 \\
FMI & 0.3356 & 0.4859 \\
NFCRMA & 0.3309 & 0.4465 \\
NFCRM1 & 0.3158 & 0.4276 \\
NFCRM2 & 0.3094 & 0.4261 \\
PSO-NFCRM & 0.2794 & 0.4042 \\
\hline
\end{tabular}

Table 6. Comparison results with $S N R=1 \mathrm{~dB}$ (Case 2).

\begin{tabular}{|c|c|c|}
\hline Algorithms & MSE $_{\text {Tr }}$ & MSE $_{\text {Ts }}$ \\
\hline \hline FCM & 2.1292 & 2.1640 \\
GK & 1.0079 & 1.3765 \\
FMI & 0.9171 & 1.1649 \\
NFCRMA & 0.9046 & 1.1395 \\
NFCRM1 & 0.8505 & 0.9491 \\
NFCRM2 & 0.8092 & 0.9194 \\
PSO-NFCRM & 0.7141 & 0.8914 \\
\hline
\end{tabular}

nace; the output $y$ is the $\mathrm{CO}_{2}$ concentration in the outlet gases. In order to take all the above-mentioned issues into account, we simulated two experimental cases: Case 1 and Case 2. In Case 1, all the 296 data pairs are used as training data and $\{y(k-1), u(k-4)\}$ are selected as input variables to NFCRM1, NFCRM2 and PSO-NFCRM algorithms. The parameter settings are $\gamma=0.01$ and $\{\gamma=1, \rho=1\}$ for the NFCRM1 and NFCRM2 algorithms, respectively. In addition, the PSO-NFCRM algorithm performs best under the following settings: $\omega=0.9$, $G=10, c_{1}=c_{2}=2, N_{P}=50$.

Figure 4 shows the modeling performance of the proposed clustering algorithms. Table 7 compares the results

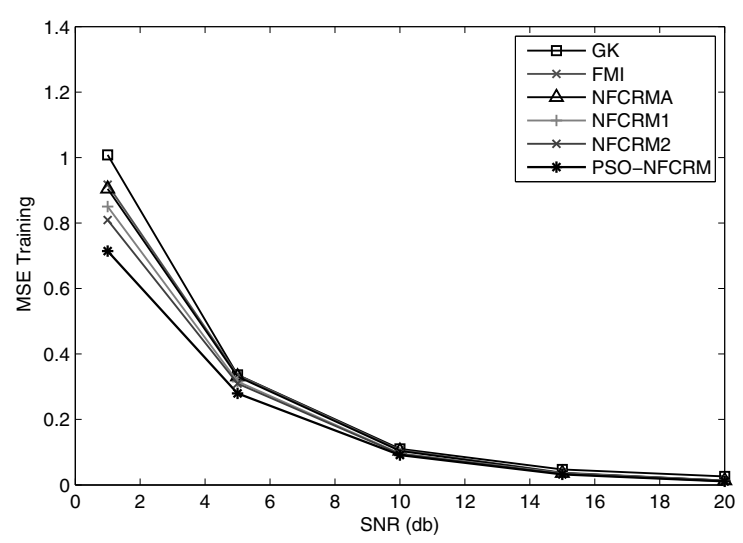

Fig. 2. MSE training performance of GK, NFCRMA, FMI, NFCRM1, NFCRM2 and PSO-NFCRM for different cases of SNR. 


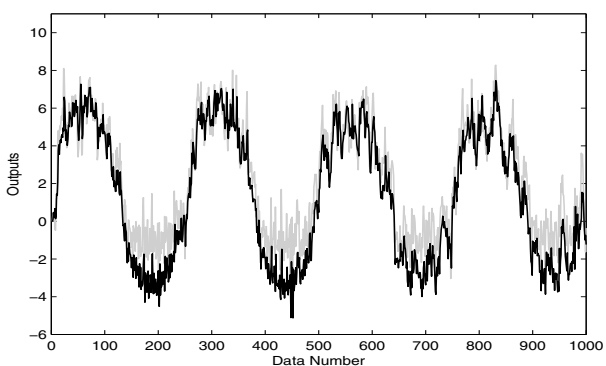

(a)

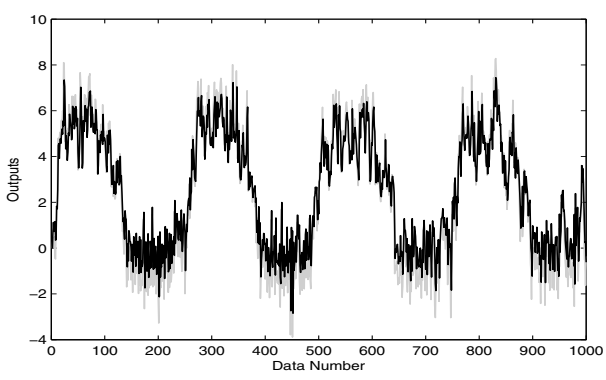

(c)

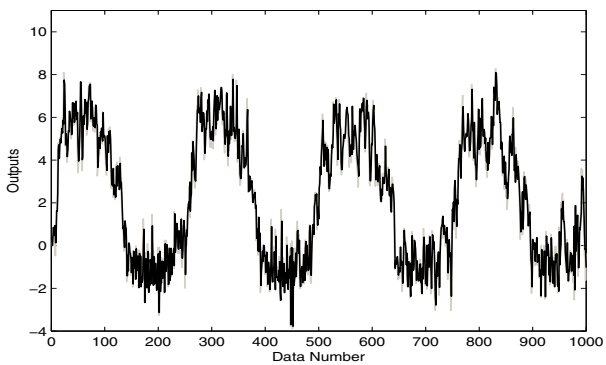

(e)

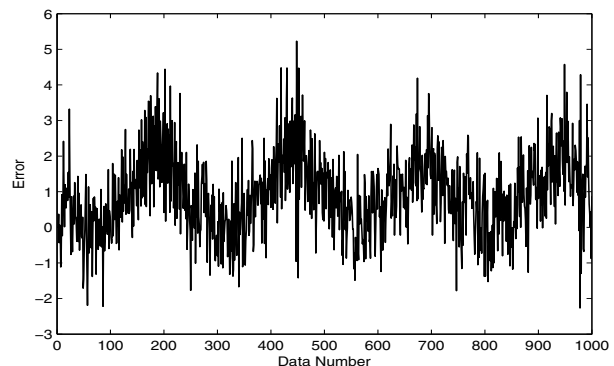

(b)

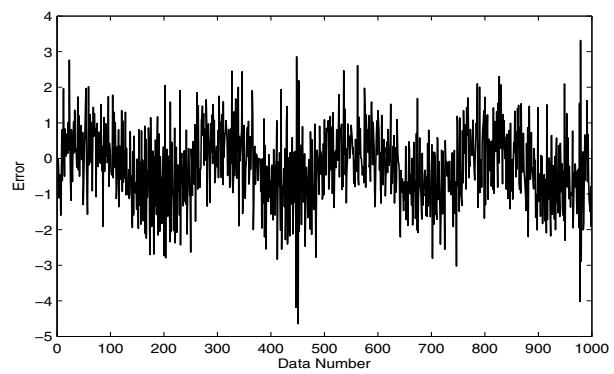

(d)

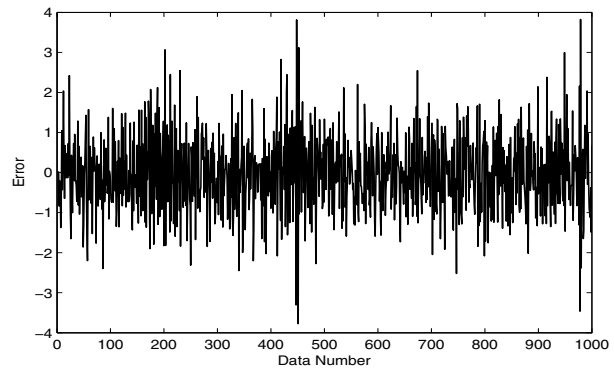

(f)

Fig. 1. FCM, NFCRMA and PSO-NFCRM performance for the testing data set with $S N R=1 \mathrm{~dB}$.

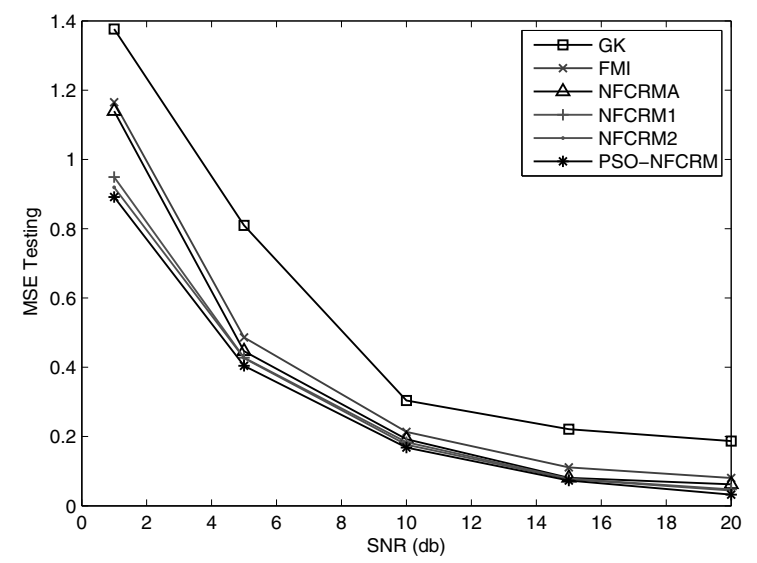

Fig. 3. MSE testing performance of GK, NFCRMA, FMI, NFCRM1, NFCRM2 and PSO-NFCRM for different cases of SNR. of the proposed models with those of other models reported by Zhang et al. (2006) as well as Andri and Ennu (2011).

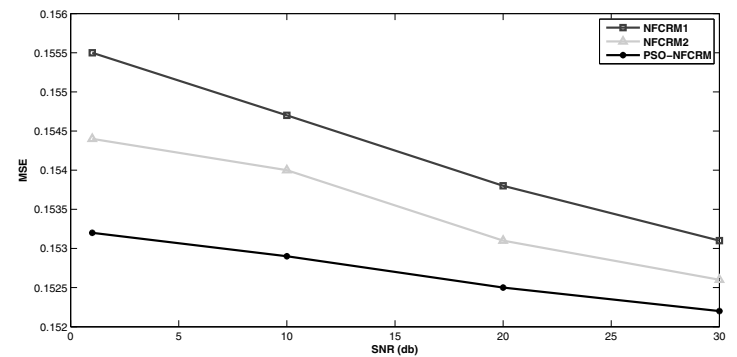

Fig. 4. MSE performance analysis of NFCRM1, NFCRM2 and PSO-NFCRM for different cases of SNR.

In Case 2, the first 148 input-output data were taken as training data and the last 148 as test data. We choose $\{y(k-1), y(k-2), y(k-3), u(k), u(k-1), u(k-2)\}$ as the variables of the fuzzy model, while the number of 
Table 7. Comparison results for gas furnace process identification (Case 1)

\begin{tabular}{|c|c|c|c|}
\hline Algorithms & $\begin{array}{c}\text { No. of } \\
\text { inputs }\end{array}$ & $\begin{array}{c}\text { No. of } \\
\text { rules }\end{array}$ & MSE \\
\hline \hline Tong (1980) & 2 & 19 & 0.469 \\
Pedrycz (1984) & 2 & 81 & 0.320 \\
Xu (1987) & 2 & 25 & 0.328 \\
Sugeno and Tanaka (1991) & 2 & 2 & 0.359 \\
Yoshinari (1993) & 2 & 6 & 0.299 \\
Joo (1997) & 2 & 6 & 0.166 \\
Chen (1998) & 2 & 3 & 0.2678 \\
Delgado (1999) & 2 & 2 & 0.396 \\
Liu (2002) & 2 & 2 & 0.1653 \\
Zhang (2006) & 2 & 2 & 0.1600 \\
Glowaty (2008) & 2 & 2 & 0.391 \\
Andri (2011) & 2 & 10 & 0.167 \\
NFCRM1 & 2 & 2 & 0.1528 \\
NFCRM1-SNR =30 dB & 2 & 2 & 0.1531 \\
NFCRM1-SNR =20 dB & 2 & 2 & 0.1538 \\
NFCRM1-SNR =10 dB & 2 & 2 & 0.1547 \\
NFCRM1-SNR =1 dB & 2 & 2 & 0.1555 \\
NFCRM2 & 2 & 2 & 0.1524 \\
NFCRM2-SNR =30 dB & 2 & 2 & 0.1526 \\
NFCRM2-SNR = 20 dB & 2 & 2 & 0.1531 \\
NFCRM2-SNR = 10 dB & 2 & 2 & 0.1540 \\
NFCRM2-SNR = 1 dB & 2 & 2 & 0.1544 \\
PSO-NFCRM & 2 & 2 & 0.1509 \\
PSO-NFCRM-SNR = 30 dB & 2 & 2 & 0.1522 \\
PSO-NFCRM-SNR = 20 dB & 2 & 2 & 0.1525 \\
PSO-NFCRM-SNR = 10 dB & 2 & 2 & 0.1529 \\
PSO-NFCRM-SNR=1 dB & 2 & 2 & 0.1532 \\
\hline
\end{tabular}

rules in our model is two. The parameter settings are $\gamma=$ 0.1 and $\{\gamma=0.1, \rho=1\}$ for NFCRM1 and NFCRM2 algorithms, respectively. In addition, the PSO-NFCRM algorithm performs best under the following settings: $\omega=$ $0.9, G=10, c_{1}=c_{2}=2, N_{P}=50$. Table 8 provides the performance of the proposed approaches compared with that of other models in the same case. The hyper-planes obtained by the PSO-NFCRM clustering algorithm are

$$
\begin{aligned}
y_{k}= & 0.8510 y_{k-1}-0.0202 y_{k-2}-0.1024 y_{k-3} \\
& -0.0398 u_{k}-0.0072 u_{k-1}-0.8256 u_{k-2} \\
& +14.4647, \\
y_{k}= & 1.2172 y_{k-1}-0.4905 y_{k-2}+0.0881 y_{k-3} \\
& +0.3219 u_{k}-0.7976 u_{k-1}-0.1038 u_{k-2} \\
& +9.8267 .
\end{aligned}
$$

Figure 5 shows a comparative MSE analysis for Case 2. It can be clearly shown that the PSO-NFCRM algorithm is more robust to noise than the NFCRM1 and NFCRM2 algorithms (Figs. 4 and 5). However, when the noise variance increases, the PSO-NFCRM clustering satisfies
Table 8. Comparison results for gas furnace process identification (Case 2).

\begin{tabular}{|c|c|c|c||}
\hline Algorithms & $\begin{array}{c}\text { No. of } \\
\text { rules }\end{array}$ & MSE $_{\mathrm{Tr}}$ & MSE $_{\mathrm{Ts}}$ \\
\hline \hline Kim et al. $(1998)$ & 2 & 0.034 & 0.244 \\
Tsekouras $(2005)$ & 2 & 0.0164 & 0.145 \\
Rezaee et al. (2010) & 2 & 0.0162 & 0.1318 \\
NFCRM1 & 2 & 0.0153 & 0.1047 \\
NFCRM1-SNR $=30 \mathrm{~dB}$ & 2 & 0.0159 & 0.1218 \\
NFCRM1-SNR $=20 \mathrm{~dB}$ & 2 & 0.0171 & 0.2317 \\
NFCRM1-SNR $=10 \mathrm{~dB}$ & 2 & 0.0180 & 0.3816 \\
NFCRM1-SNR $=1 \mathrm{~dB}$ & 2 & 0.0193 & 0.4414 \\
NFCRM2 & 2 & 0.0151 & 0.0955 \\
NFCRM2-SNR $=30 \mathrm{~dB}$ & 2 & 0.0153 & 0.0961 \\
NFCRM2-SNR $=20 \mathrm{~dB}$ & 2 & 0.0165 & 0.1639 \\
NFCRM2-SNR $=10 \mathrm{~dB}$ & 2 & 0.0172 & 0.2030 \\
NFCRM2-SNR $=1 \mathrm{~dB}$ & 2 & 0.0189 & 0.2208 \\
PSO-NFCRM & 2 & 0.0148 & 0.0937 \\
PSO-NFCRM-SNR $=30 \mathrm{~dB}$ & 2 & 0.0151 & 0.0952 \\
PSO-NFCRM-SNR = 20 dB & 2 & 0.0155 & 0.1546 \\
PSO-NFCRM-SNR $=10 \mathrm{~dB}$ & 2 & 0.0161 & 0.1887 \\
PSO-NFCRM-SNR = 1 dB & 2 & 0.0183 & 0.2084 \\
\hline
\end{tabular}

the convergence conditions. Consequently, the PSONFRCM algorithm becomes more robust to noise with MSE $=0.0183$. The other two algorithms, NFRCM1 and NFCRM2, exhibit poor performance with the MSE equal to 0.0193 and 0.0189 , respectively. A similar analysis can be seen also in Table 7. In the absence of noise, it is clear that PSO-NFCRM performs better than the other algorithms reported in the literature (Tables 7 and 8 ). A good approximation accuracy of PSO-NFCRM is shown in Fig. 5.

\section{Conclusions}

In this paper, a new fuzzy c-regression clustering algorithm is proposed using a modified objective function, a new error measure, and a parameter estimation based particle swarm optimization. The application of a modified objective function improves the robustness of the FCRM method, based on the noise clustering algorithm and the new error measure. Yet, the PSO procedure allows achieving the global minimum of the new objective function. The proposed modifications of the FCRM method provide better results of fuzzy modeling of unknown nonlinear systems. The robustness and the quality of these modifications in the FCRM method are demonstrated by simulation results of two benchmark problems. Thus, the proposed methods show favorable results compared with the techniques reported in the literature. Consequently, they may be extended to identification of complex applications regarding various types of modeling problems in 


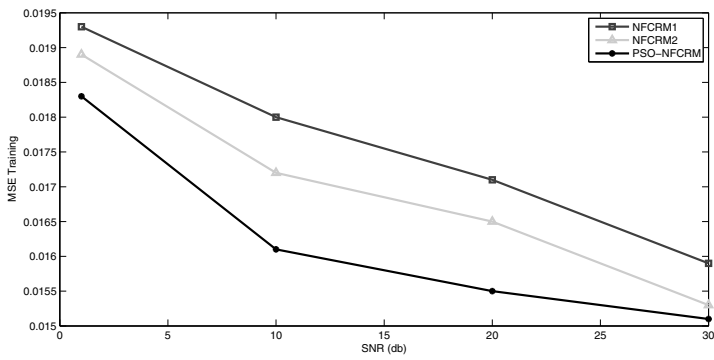

(a)

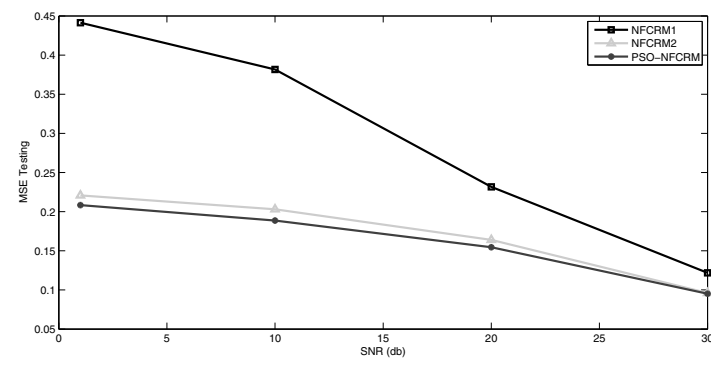

(b)

Fig. 5. MSE training and testing performance analysis of NFCRM1, NFCRM2 and PSO-NFCRM for different cases of SNR.

noisy environments.

\section{Acknowledgment}

The authors would like to express their gratitude to Mrs. Rabeb Saidi for linguistic advice.

\section{References}

Alci, M. (2008). Fuzzy rule-base driven orthogonal approximation, Neural Computing and Applications 17(5-6): 501507.

Andri, R. and Ennu, R. (2011). Identification of transparent, compact, accurate and reliable linguistic fuzzy models, Information Sciences 181(20): 4378-4393.

Ben, N., Yunlong, Z., Xiaoxian, H. and Hai, S. (2008). A multi-swarm optimizer based fuzzy modeling approach for dynamic systems processing, Neurocomputing 71(79): $1436-1448$.

Bezdek, J.C. (1981). Pattern Recognition with Fuzzy Objective Function Algorithms, Plenum Press, New York, NY.

Bezdek, J.C., Keller, J., Krisnapuram, R. and Pal, N. (1999). Fuzzy Models and Algorithms for Pattern Recognition and Image Processing, Vol. 4, Springer, New York, NY.

Bidyadhar, S. and Debashisha, J. (2011). A differential evolution based neural network approach to nonlinear identification, Applied Soft Computing 11(1): 861-871.

Boukhris, A., Mourot, G. and Ragot, J. (1999). Nonlinear invisible system identification: A multi-model approach, International Journal of Control 72(7-8): 591-604.
Box, G.E.P. and Jenkins, G.M. (1970). Times Series Analysis, Holden Day, San Francisco, CA.

Brdyś, A.M. and Littler, J.J. (2002). Fuzzy logic gain scheduling for non-linear servo tracking, International Journal of Applied Mathematics and Computer Science 12(2): 209-219.

Celikyilmaz, A. and Burhan Turksen, I. (2008). Enhanced fuzzy system models with improved fuzzy clustering algorithm, IEEE Transactions on Fuzzy Systems 16(3): 779-794.

Chaoshun, L., Jianzhong, Z., Xiuqiao, X., Qingqing, L. and Xueli, A. (2009). T-S fuzzy model identification based on a novel fuzzy c-regression model clustering algorithm, Engineering Applications of Artificial Intelligence $\mathbf{2 2}$ (4-5): 646-653.

Chaoshun, L., Jianzhong, Z., Xiuqiao, X., Qingqing, L. and Xueli, A. (2010). A new T-S fuzzy-modeling identification approach to identify a boiler-turbine, Expert Systems with Applications 37(3): 2214-2221.

Chen, J.L. and Wang, J.H. (1999). A new robust clustering algorithm-density-weighted fuzzy c-means, Proceedings of the IEEE Conference on Systems, Man, and Cybernetics, SMC 1999, Tokyo, Japan, pp. 12-15.

Chen, J.Q., Xi, Y.G. and Zhang, Z.J. (1998). A clustering algorithm for fuzzy model identification, International Journal of Control 98(3): 319-329.

Chen, S., Billings, S.A. and Luo, W. (1989). Orthogonal least squares methods and their application to nonlinear system identification, International Journal of Control 50(5): 1873-1896.

Dave, R.N. (1991). Characterization and detection of noise in clustering, Pattern Recognition Letters 12(11): 657-664.

Dave, R.N. and Krishnapuram, R. (1997). Robust clustering methods: A unified view, IEEE Transactions on Fuzzy Systems 5(2): 270-293.

Frigui, H. and Krishnapuram, R. (1999). A robust competitive clustering algorithm with applications in computer vision, IEEE Transactions on Pattern Analysis and Machine Intelligence 21(5): 450-465.

Gath, I. and Geva, A. (1989). Unsupervised optimal fuzzy clustering, IEEE Transactions on Pattern Analysis and Machine Intelligence 11(7): 773-780.

Gustafson, D.E. and Kessel, W.C. (1979). Fuzzy clustering with a fuzzy covariance matrix, Proceedings of the IEEE Conference on Decision Control, CDC 1978, San Diego, CA, USA, pp. 761-766.

Hathaway, R.J. and Bezdek, J.C. (1993). Switching regression models and fuzzy clustering, IEEE Transactions on Pattern Analysis and Machine Intelligence 1(3): 195-204.

Hellendoorn, H. and Driankov, D. (1997). Fuzzy Model Identification: Selected Approaches, Springer, Berlin.

Honda, K., Notsu, A. and Ichihashi, H. (2010). Fuzzy PCAguided robust k-means clustering, IEEE Transactions on Pattern Analysis and Machine Intelligence 18(1): 67-79.

Hoppner, F., Klawonn, F., Kruse, R. and Runkler, T. (1999). Fuzzy Cluster Analysis, Methods for Classification, Data Analysis and Image Recognition, 1st Edn., John Wiley and Sons, Chichester. 
Ichalal, D., Marx, B., Ragot, J. and Maquin, D. (2010). Observer based fault tolerant control for nonlinear TakagiSugeno systems: An LMI approach, Proceedings of the 18th Mediterranean Conference on Control and Automation, MED 2010, Marrakech, Marocco, pp. 1278-1283.

Ichihashi, H. and Honda, K. (2004). On parameter setting in applying Dave's noise fuzzy clustering to Gaussian mixture models, Proceedings of the 13th IEEE International Conference on Fuzzy Systems, FUZZ-IEEE 2004, Budapest, Hungary, pp. 1501-1506.

Ichihashi, H., Honda, K. and Wakami, N. (2005). Robust PCA with intra-sample outlier process based on fuzzy Mahalanobis distances and noise clustering, Proceedings of the 14th IEEE International Conference on Fuzzy Systems, FUZZ-IEEE 2005, Reno, NV, USA, pp. 640-645.

Kennedy, J. and Eberhart, R. C. (1995). Particle swarm optimization, Proceedings of the IEEE International Conference on Neural Networks, ICNN 1995, Perth, Australia, pp. 19421948.

Kim, E., Park, M., Kim, S. and Park, M. (1998). A transformed input-domain approach to fuzzy modeling, IEEE Transactions on Fuzzy Systems 6(4): 596-604.

Kim, K., Kim, Y.K., Kim, E. and Park, M. (2004). A new TSK fuzzy modeling approach, Proceedings of the IEEE International Conference on Fuzzy Systems, FUZZ-IEEE 2004, Budapest, Hungary, pp. 773-776.

Kluska, J. (2009). Analytical Methods in Fuzzy Modeling and Control, Studies in Fuzziness and Soft Computing, Springer-Verlag, Berlin/Heidelberg.

Kościelny, J.M. and Syfert, M. (2006). Fuzzy diagnostic reasoning that takes into account the uncertainty of the relation between faults and symptoms, International Journal of Applied Mathematics and Computer Science 16(1): 27-35.

Leski, J.M. (2004). $\epsilon$-insensitive fuzzy c-regression models: Introduction to $\epsilon$-insensitive fuzzy modeling, IEEE Transactions on Systems, Man, and Cybernetics 34(1): 4-15.

Liang, Z., Yang, Y. and Zeng, Y. (2009). Eliciting compact T-S fuzzy models using subtractive clustering and coevolutionary particle swarm optimization, Neurocomputing 72(1012): $2569-2575$.

Marx, B., Koenig, D. and Ragot, J. (2007). Design of observers for Takagi-Sugeno descriptor systems with unknown inputs and application to fault diagnosis, IET Control Theory and Applications 1(5): 1487-1495.

Nasraoui, O. and Krishnapuram, R. (1996). An improved possibilistic c-means algorithm with finite rejection and robust scale estimation, Biennial Conference of the North American Fuzzy Information Processing Society, NAFIPS 1996, Berkeley, CA, USA, pp. 395-399.

Niknam, T. and Amiri, B. (2010). An efficient hybrid approach based on PSO, ACO and k-means for cluster analysis, $A p$ plied Soft Computing 10(1): 183-197.

Ohashi, Y. (1984). Fuzzy clustering and robust estimation, 9th Meeting, SAS Users Group International, Hollywood Beach, FL, USA, pp. 1-6.
Panchal, V.K., Harish, K. and Jagdeep, K. (2009). Comparative study of particle swarm optimization based unsupervised clustering techniques, International Journal of Computer Science and Network Security 9(10): 132-140.

Qi, R. and Brdys, M.A. (2009). Indirect adaptive controller based on a self-structuring fuzzy system for nonlinear modeling and control, International Journal of Applied Mathematics and Computer Science 19(4): 619-630, DOI: 10.2478/v10006-009-0049-8.

Qiang, N. and Xinjian, H. (2011). An improved fuzzy c-means clustering algorithm based on PSO, Journal of Software 6(5): $873-879$.

Rezaee, B. and Zarandi, M.H.F. (2010). Data-driven fuzzy modeling for Takagi-Sugeno-Kang fuzzy system, Information Sciences 180(2): 241-255.

Soltani, M., Aissaoui, B., Chaari, A., Ben Hmida, F. and Gossa, M. (2011). A modified fuzzy c-regression model clustering algorithm for T-S fuzzy model identification, Proceedings of the 8th IEEE International Multi-Conference on Systems, Signals and Devices, SSD 2011, Sousse, Tunisia, pp. 1-6.

Soltani, M., Chaari, A., Ben Hmida, F. and Gossa, M. (2010a). Modified fuzzy model identification clustering algorithm for liquid level process, Proceedings of the 18th Mediterranean Conference on Control and Automation, MED 2010, Marrakech, Morocco, pp. 1151-1157.

Soltani, M., Chaouchi, L., Chaari, A., Ben Hmida, F. and Moncef, G. (2010b). Identification of nonlinear complex systems using uncoupled state fuzzy model for liquid level process, International Review of Automatic Control 3(5): 535-544.

Sumit, S. and Dave, R.N. (1998). Clustering of relational data containing noise and outliers, Proceedings of the 7 th IEEE International Conference on Fuzzy Systems/World Congress on Computational Intelligence, Anchorage, AK, USA, Vol. 2, pp. 1411-1416.

Takagi, T. and Sugeno, M. (1985). Fuzzy identification of systems and its application to modeling and control, IEEE Transactions on Systems, Man, and Cybernetics 15(1): 116-132.

Tran, D. and Wagner, M. (1999). A robust clustering approach to fuzzy gaussian mixture models for speaker identification, Proceedings of the 3rd International Conference on Knowledge-Based Intelligent Information Engineering Systems, KES 1997, Adelaide, SA, Australia, pp. 337-340.

Wang, L.X. and Mendel, J.M. (1992). Fuzzy basis functions, universal approximation, and orthogonal leastsquares learning, IEEE Transactions on Neural Networks 3(5): 807-814.

Wu, K.L. and Yang, M.S. (2002). Alternative c-means clustering algorithms, Pattern Recognition 35(10): 2267-2278.

Wu, X.F., Lang, Z.Q. and Billings, S.A. (2005). An orthogonal least squares based approach to FIR designs, International Journal of Automation and Computing 2(2): 163-170. 
Xu, Y.F. and Zhang, S.L. (2009). Fuzzy particle swarm clustering of infrared images, Proceedings of the 2009 2nd International Conference on Information and Computing Science, ICIC 2009, Manchester, UK, Vol. 2, pp. 122-124.

Yang, X., Song, Q. and Liu, S. (2005). Robust deterministic annealing algorithm for data clustering, Proceedings of the International Joint Conference on Neural Networks, IJCNN 2005, Montreal, Canada, pp. 1878-1882.

Ying, H. (2000). Fuzzy Control and Modeling: Analytical Foundations and Applications, IEEE Press, New York, NY.

Ying, K.C., Lin, S.W., Lee, Z.J. and Lee, I.L. (2011). A novel function approximation based on robust fuzzy regression algorithm model and particle swarm optimization, Applied Soft Computing 38(2): 1820-1826.

Zhang, D., Liu, X. and Guan, Z. (2006). A dynamic clustering algorithm based on PSO and its application in fuzzy identification, Proceedings of the International Conference on Intelligent Information Hiding and Multimedia Signal Processing, IIH-MSP 2006, Pasadena, CA, USA, pp. 232-235.

Zhang, Y., Huang, D., Ji, M. and Xie, F. (2011). Image segmentation using PSO and PCM with Mahalanobis distance, Expert Systems with Applications 38(7): 9036-9040.
Moêz Soltani was born in 1980, Tunisia. He is a Ph.D. graduate student. He obtained his B.Sc. and M.Sc. degrees in electrical engineering in 2004 and 2006, respectively, from the High School of Sciences and Engineering of Tunis (ESSTT), Tunisia. His main research interests are fuzzy logic and its application in the identification and control of nonlinear systems.

Abdelkader Chaari was born in 1957. He is an associate professor at the High School of Sciences and Engineering of Tunis (ESSTT). He obtained his H.D.R. degree in 2008 from the same school. His main research interests are the identification and control of nonlinear systems, robust estimation and robust filtering, and fuzzy logic.

Fayçal Ben Hmida was born in 1966. He is an associate professor at the High School of Sciences and Engineering of Tunis (ESSTT). He obtained his M.Sc. in 1992 and Ph.D. in 1996 from Aix-Marseille III University, France, and the H.D.R. in 2011 from the High School of Sciences and Engineering of Tunis. His main research interests are fault detection and isolation, robust estimation and robust filtering, and fuzzy logic.
Received: 5 August 2011

Revised: 9 January 2012

Re-revised: 21 April 2012 